\title{
A ressonância magnética do joelho usando a bobina de corpo é equivalente à TC na medição da distância TT- ST: Removendo o viés sistemático*
}

\section{Knee MR Using a Body Coil is Equivalent to CT in Measuring the TT-TG Distance: Removing the Systematic Bias}

\author{
Laís Uyeda Aivazoglou ${ }^{10}$ Mariana Kei Toma1ํำ Pedro Henrique Coelho Arruda ${ }^{10}$ \\ Alipio Gomes Ormond Filho ${ }^{10}$ Julio Brandão Guimarães ${ }^{10}$ Flávio Duarte Silva1(1) \\ ${ }^{1}$ Departamento de Radiologia, Grupo Fleury Medicina e Saúde, São
Paulo, SP, Brasil \\ Rev Bras Ortop 2022;57(1):82-88 \\ Endereço para correspondência Laís Uyeda Aivazoglou, MD, \\ Department of Radiology, Grupo Fleury Medicina e Saúde, Rua Mato \\ Grosso, 306, SL1, São Paulo, SP, 01239-040, Brasil \\ (e-mail: lais.aivazoglou@grupofleury.com.br).
}

\section{Resumo \\ Palavras-chave \\ - joelho \\ - imagem de ressonância magnética \\ - instabilidade patelar \\ - tomografia computadorizada de raios $\mathrm{x}$}

Objetivo Comparar a ressonância magnética (RM) usando uma bobina corporal e tomografia computadorizada (TC) na medição da distância tubérculo tibial-sulco troclear (TT-ST) e as distâncias tendão patelar-sulco troclear cartilaginoso (TP-STC), e avaliar a confiabilidade interavaliador.

Métodos O grupo de estudo consistiu em 34 joelhos de 17 indivíduos assintomáticos sem história de patologia, trauma ou cirurgia no joelho. Uma tomografia computadorizada (TC) de baixa dose e uma sequência axial de RM ponderada em T1 dos joelhos foram realizadas com padronização rigorosa do posicionamento com extensão total dos joelhos e pés paralelos. Dois radiologistas musculoesqueléticos realizaram as medidas de forma independente. A confiabilidade das distâncias TT-ST e TP-STC na TC $(17,1 \pm 4,2 \mathrm{~mm}$ e $17,3 \pm 4,2 \mathrm{~mm})$ e RM $(16,2 \pm 3,7 \mathrm{~mm}$ e $16,5 \pm 4,1 \mathrm{~mm})$, foi avaliada pelo coeficiente de correlação intraclasse (CCl $[2,1)]$ ) e gráficos Bland-Altman, bem como a confiabilidade entre avaliadores para ambos os métodos.

Resultados Boa confiabilidade e concordância foram observadas entre as medidas de TC e RM para TT-ST e TP-STC com um CCI de $0,774(p<0,001)$ e $0,743(p<0,001)$, respectivamente, e nenhum viés sistemático foi observado. A confiabilidade entre avaliadores foi excelente para todas as medições em ambos os métodos de imagem. Conclusão Este foi o primeiro estudo que comparou a RM usando a bobina de corpo com a TC na medição da distância TT-ST, com a implicação clínica potencial de que a TC neste cenário clínico poderia ser evitada.

\footnotetext{
Trabalho desenvolvido no Departamento de Radiologia, Grupo Fleury Medicina e Saúde, São Paulo, SP, Brasil.
}

recebido 24 de Abril de 2020 aceito 06 de Julho de 2020 Publicado on-line Março 22, 2021
DOI https://doi.org/ $10.1055 / \mathrm{s}-0040-1718511$ ISSN 0102-3616.
(C) 2021. Sociedade Brasileira de Ortopedia e Traumatologia. All rights reserved.

This is an open access article published by Thieme under the terms of the Creative Commons Attribution-NonDerivative-NonCommercial-License, permitting copying and reproduction so long as the original work is given appropriate credit. Contents may not be used for commercial purposes, or adapted, remixed, transformed or built upon. (https://creativecommons.org/ licenses/by-nc-nd/4.0/)

Thieme Revinter Publicações Ltda., Rua do Matoso 170, Rio de Janeiro, RJ, CEP 20270-135, Brazil 


\author{
Abstract \\ Keywords \\ - knee \\ - magnetic resonance \\ imaging \\ - patellar instability \\ - tomography, \\ $\mathrm{x}$-ray computed
}

Objective To compare magnetic resonance imaging (MRI) using a body coil with computed tomography $(\mathrm{CT})$ in measuring the tibial tubercle-trochlear groove distance (TT-TG) and the patellar tendon-cartilaginous trochlear groove (PT-CTG) distances, and evaluate interrater reliability.

Methods The study group consisted of 34 knees from 17 asymptomatic subjects with no history of knee pathology, trauma or surgery. A low-dose CT scan and an axial T1weighted MRI sequence of the knees were performed with rigorous standardization of the positioning with full extension of the knees and parallel feet. Two musculoskeletal radiologists performed the measurements independently. The reliability of the TT-TC and PT-CTG distances on CT $(17.1 \pm 4.2 \mathrm{~mm}$ and $17.3 \pm 4.2 \mathrm{~mm})$ and of MRI $(16.2 \pm 3.7 \mathrm{~mm}$ and $16.5 \pm 4.1 \mathrm{~mm})$ was assessed by intraclass correlation coefficient (ICC $[2,1]$ ) and Bland-Altman graphs, as well as the interrater reliability for both methods.

Results Good reliability and agreement was observed between CT and MRI measurements for TT-TG and PT-CTG, with an ICC of $0.774(p<0.001)$ and $0.743(p<0.001)$, respectively, and no systematic bias was observed. The interrater reliability was excellent for all measurements on both imaging methods.

Conclusion This was the first study that compared MRI using a body coil with $\mathrm{CT}$ in measuring the TT-TG distance, with the potential clinical implication that the CT in this clinical setting could be avoided.

\section{Introdução}

A instabilidade patelar representa uma condição de saúde comum e significativa que afeta jovens e pode levar à osteoartrite precoce, ${ }^{1}$ com uma incidência de até $12,98 /$ 100.000 pessoas-ano em homens entre 15 e 19 anos de idade. $^{2}$ Sua etiologia é multifatorial, exigindo diagnóstico preciso, pois as opções de tratamento variam de terapias conservadoras a diferentes intervenções cirúrgicas. ${ }^{3}$

Um dos fatores de risco mais reconhecidos para instabilidade patelar é o aumento da distância do tubérculo tibial ao sulco troclear (TT-ST); quando maior que 15 a $20 \mathrm{~mm}$, é geralmente considerada patológica e foi proposta como um limite para considerar uma osteotomia do tubérculo tibial ou procedimento de realinhamento distal. ${ }^{4,5} \mathrm{O}$ método de imagem padrão ouro para realizar esta medição é a tomografia computadorizada (TC), que se mostrou confiável. ${ }^{6}$ Por outro lado, muitos dos pacientes com instabilidade patelar são submetidos à ressonância magnética (RM) do joelho para avaliar lesões de tecidos moles, como rupturas ligamentares e defeitos condrais; portanto, seria desejável avaliar de forma confiável a distância TT-ST no mesmo estudo de imagem e, assim, evitar a necessidade de um estudo de TC extra. Além disso, muitos pacientes com instabilidade patelar são jovens e evitar a exposição à radiação seria consideravelmente benéfico.

Muitos autores descobriram que as medidas de TC e RM para TT-ST não são equivalentes, e que as medidas de RM são sistematicamente menores, ${ }^{3,4,7-9}$ o que sugere que seria impreciso usar o mesmo limiar em RM e TC no diagnóstico e planejamento cirúrgico.
A distância TT-ST é altamente sensível a mudanças no posicionamento do joelho $0^{4,10}$ e enquanto a TC é realizada com as pernas em extensão total, a bobina de joelho dedicada na RM envolve o joelho de uma forma que este assume graus variáveis de flexão $\left(\sim 25^{\circ}\right)$ e desvio em varo. ${ }^{4} \mathrm{~A}$ literatura é escassa e controversa sobre a influência do posicionamento dos pés nas medidas do joelho.

Outro tópico controverso na literatura é quais marcos usar para a medição da distância TT-ST. Dada a alta resolução de contraste de partes moles da RM, alguns autores usaram parâmetros de partes moles em vez de parâmetros ósseos para medir a lateralização do tubérculo tibial: o nadir do sulco troclear cartilaginoso (STC) em vez do sulco troclear ósseo e a inserção tibial do tendão patelar (TP) em vez do tubérculo tibial (TT).

Até esta data, nenhum estudo comparou a medição TT-ST e TP-STC em RM usando uma bobina corporal com TC para testar a equivalência. Portanto, os objetivos do presente estudo são comparar esses valores de medidas entre a RM usando bobina de corpo com a TC em voluntários assintomáticos, e avaliar a concordância interobservadores e entre os métodos. Nossa hipótese é que garantindo o mesmo posicionamento de joelho e pés na RM e na TC, as medidas resultariam semelhantes.

\section{Métodos}

A aprovação do comitê de ética foi obtida (Plataforma Brasil número 3136833), bem como consentimento informado de todos os participantes. $\mathrm{O}$ tamanho da amostra foi calculado de acordo com Zou, ${ }^{11}$ considerando um tamanho de efeito de 
0,65 , um nível de significância bicaudal $(\alpha)$ de $5 \%$ e uma força ( $\beta$ ) de $80 \%$. Este cálculo resultou em um mínimo de 30 joelhos.

Voluntários sem quaisquer sintomas clínicos no joelho foram inscritos. O grupo de estudo foi composto por 34 joelhos (17 indivíduos; 13 homens e 4 mulheres), com média de idade e desvio padrão (DP) de $38,6 \pm 6,4$ anos, variando entre 29 e 50 anos. 0 critério de inclusão foi a ausência de sintomas no joelho e os critérios de exclusão foram: cirurgia anterior no joelho, trauma prévio no joelho, história de instabilidade patelar ou qualquer outra patologia conhecida no joelho.

Uma TC de baixa dose e uma sequência de RM axial ponderada em T1 dos joelhos foram realizadas em todos os indivíduos. O posicionamento foi o mesmo nos dois estudos: os voluntários foram escaneados em decúbito dorsal com extensão total dos joelhos, por meio de um dispositivo de suporte de acrílico que mantinha a orientação das faces mediais dos pés paralelas entre si, com distância de 3 a $5 \mathrm{~cm}$ entre eles (-Fig. $\mathbf{1}$ ).

Os estudos de TC foram realizados em um scanner de TC Siemens (SOMATOM Definition Edge, Siemens Medical Solutions, Munique, Alemanha) de 64 detectores e o sistema de controle CARE Dose foi selecionado para atingir a redução da dose de radiação. Para a avaliação do comitê de ética, realizamos o cálculo da dose de radiação em fantomas padrão e a dose efetiva resultou em $\sim 0,01 \mathrm{mSv}$ (metade da dose de uma radiografia de tórax posteroanterior). ${ }^{12}$ As imagens foram reformatadas para $3 \mathrm{~mm}$ de espessura usando tecidos moles e janelas ósseas. Os estudos de RM foram realizados em um scanner de ressonância magnética GE/
Optima 450w 1,5T (GE, Boston, MA, EUA) com a bobina de corpo e consistiu em uma sequência ponderada em T1 axial (TR: $375 \mathrm{~ms} / \mathrm{TE}: 8,32 \mathrm{~ms}$ ) de ambos os joelhos, $5 \mathrm{~mm}$ de espessura, espaçamento de $1 \mathrm{~mm}$, matriz $320 \times 256$. Além disso, ambos os exames incluíram a tróclea femoral e a tuberosidade tibial para permitir medições.

Após uma sessão de treinamento, dois radiologistas musculoesqueléticos certificados (5 e 2 anos de experiência) avaliaram as imagens de TC e RM de forma independente e escolheram estes cortes específicos:

1. Corte mais cranial que retratava a cobertura cartilaginosa completa da tróclea femoral na RM e na TC (janela de partes moles e osso), permitindo a determinação do ponto mais profundo do sulco da tróclea óssea (ST) e do sulco da tróclea cartilaginosa (STC);

2. O corte que mostrava a inserção completa do tendão patelar na tuberosidade tibial na RM e TC (janela de partes moles), e o ponto médio dessa êntese foi definido como o ponto de referência do TP;

3. O ponto mais anterior da tuberosidade tibial na RM e na TC (janela óssea), que correspondia à marca óssea do TT.

Após esta primeira sessão independente, como nosso principal interesse era estudar a relação entre a posição do joelho e as medidas, quaisquer diferenças nos cortes escolhidos foram corrigidas por consenso. Em seguida, eles foram sobrepostos e as distâncias TT-ST e TP-STC foram medidas independentemente em ambos os métodos (-Fig. 2). A distância TT-ST foi avaliada entre duas linhas paralelas traçadas através dos parâmetros ósseos: o ponto mais
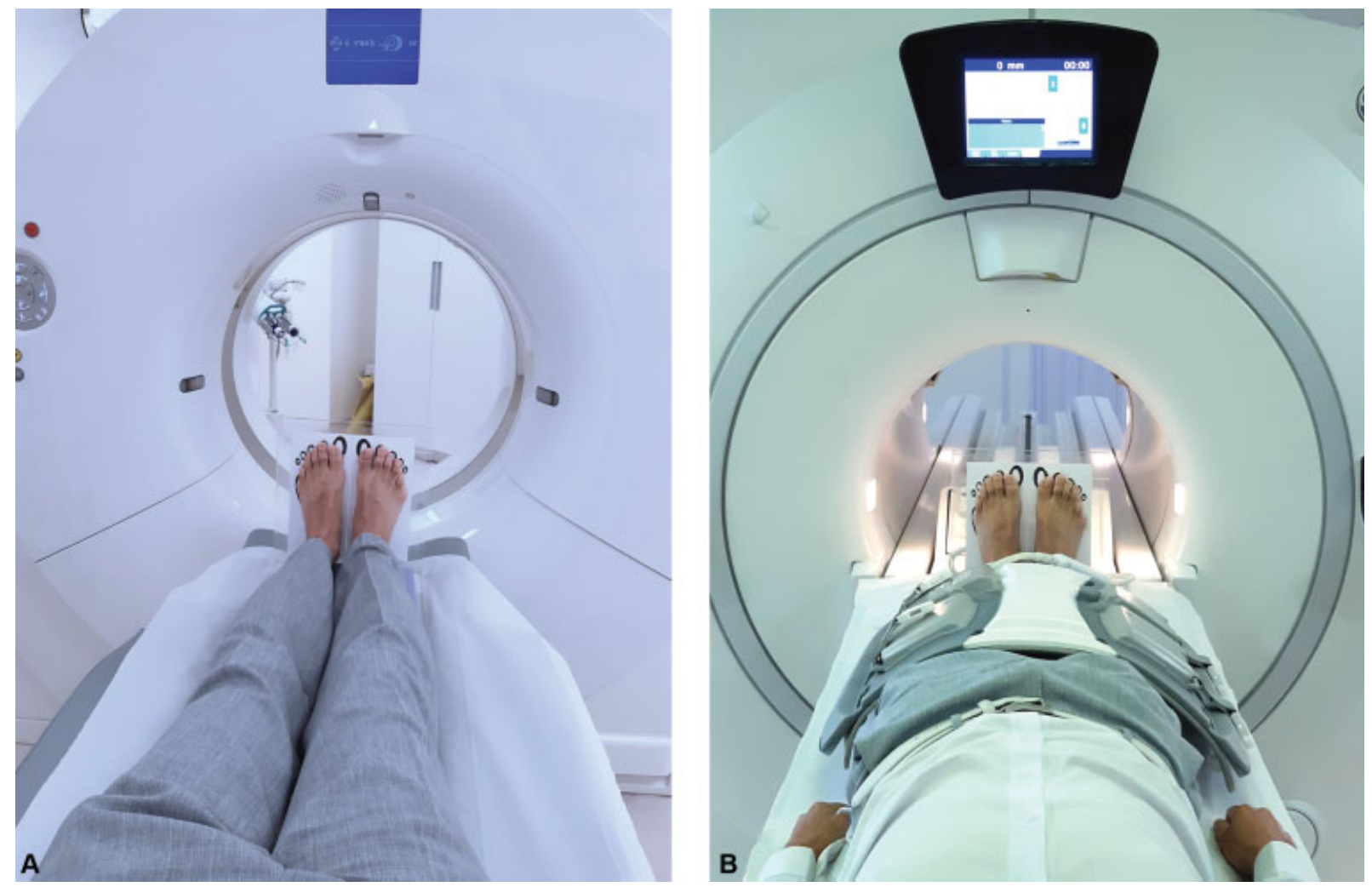

Fig. 1 (A e B) Posicionamento em TC e RM com bobina corporal, ambas com dispositivo de suporte de acrílico. 


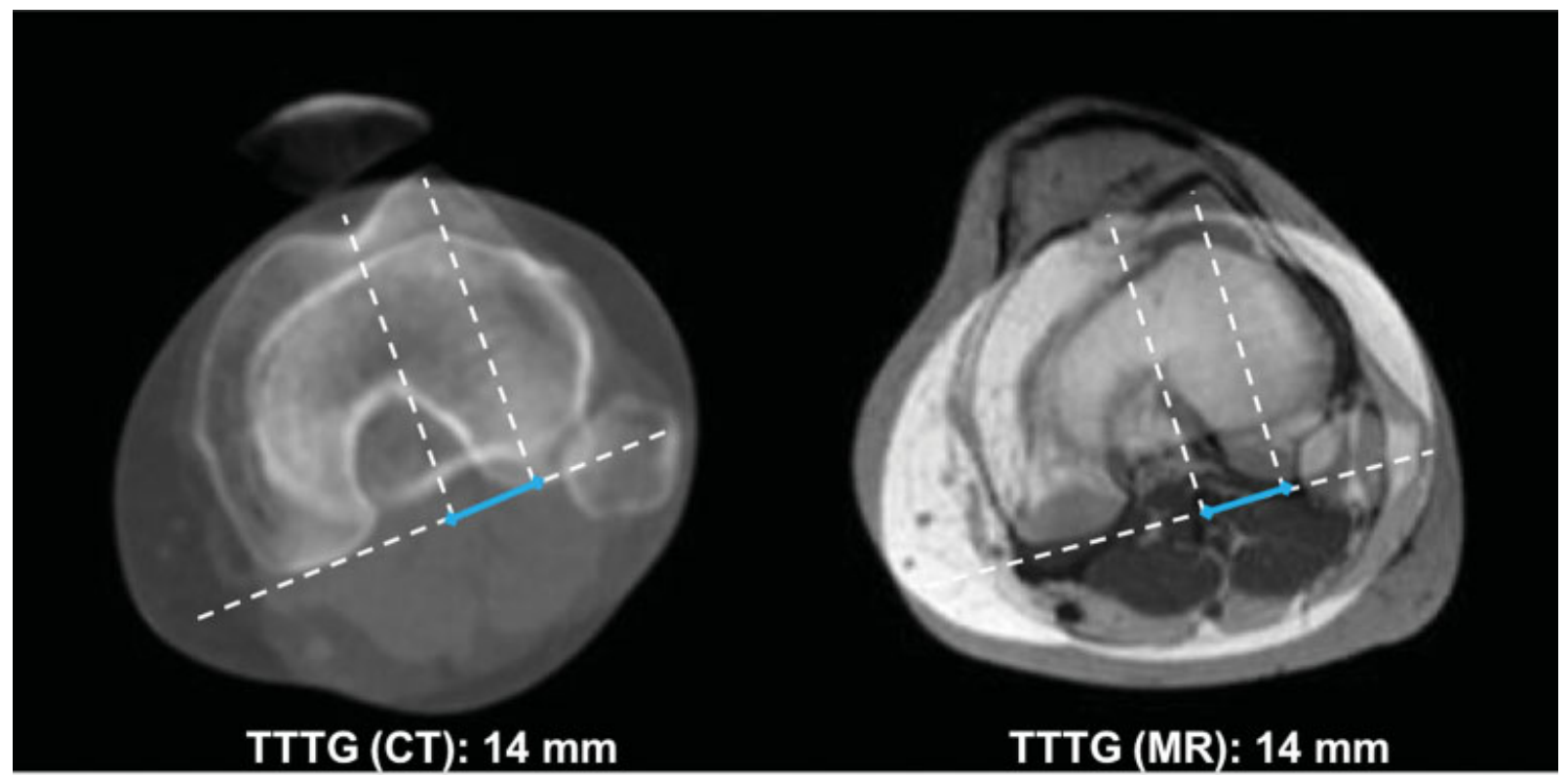

Fig. 2 A imagem mostra os cortes de TC (esquerda) e RM (direita) sobrepostos e mostra a medição TT-ST no joelho esquerdo de uma voluntária assintomática de 29 anos de idade.

anterior da tuberosidade tibial e o ponto mais profundo do sulco troclear ósseo, perpendiculares a uma linha desenhada tangente à face posterior dos côndilos femorais. ${ }^{13}$ A distância TP-STC foi medida de forma semelhante, mas usando os parâmetros de partes moles: a inserção do TP à tíbia e o ponto mais profundo do STC. ${ }^{14}$

A análise estatística foi feita usando SPSS Statistics for Windows, Versão 20.0 (IBM Corp., Armonk, NY, EUA), STATA 12 (Stata Software, College Station, TX, EUA) e o software R(R Foundation, Viena, Áustria). A distribuição de normalidade foi avaliada pelo teste Kolmogorov-Smirnov. A confiabilidade entre avaliadores dos cortes escolhidos, as medições TT-ST e TP-STC na TC e na RM foram avaliados para todas as medidas estudadas usando o coeficiente de correlação intraclasse (CCI $[2,1])$ e gráficos de Bland-Altman. O tipo de CCI escolhido foi baseado nas diretrizes de Koo et al. ${ }^{15}$ e foi adotado o nível de significância $(\alpha)=0,05$.

Tabela 1 Confiabilidade entre avaliadores para cada fatia

\begin{tabular}{|l|l|l|l|}
\hline corte & CCI (2,1) (IC 95\%) & $\begin{array}{l}\text { mesmo } \\
\text { corte }\end{array}$ & $\begin{array}{l}\text { o mesmo ou o } \\
\text { próximo corte }\end{array}$ \\
\hline 1 na TC & $0,993(0,985-0,997)$ & $73,5 \%$ & $97,1 \%$ \\
\hline 2 na TC & $0,996(0,989-0,998)$ & $64,7 \%$ & $100 \%$ \\
\hline 3 na TC & $0,989(0,928-0,996)$ & $32,4 \%$ & $76,5 \%$ \\
\hline 1 na RM & $0,967(0,934-0,984)$ & $70,6 \%$ & $100 \%$ \\
\hline 2 na RM & $0,896(0,796-0,947)$ & $64,7 \%$ & $94,1 \%$ \\
\hline 3 na RM & $0,961(0,882-0,984)$ & $52,9 \%$ & $91,2 \%$ \\
\hline
\end{tabular}

Abreviações: $\mathrm{CCl}$, coeficiente de correlação intraclasse; IC, intervalo de confiança; RM, ressonância magnética; TC, tomografia computadorizada.

\section{Resultados}

A normalidade avaliada pelo teste de Kolmogorov-Smirnov sendo a hipótese nula de uma distribuição normal resultou em um $p>0,05$ para todas as variáveis. O CCI para todos os cortes escolhidos foi excelente, exceto para o TP na RM, que foi bom (-Tabela 1). A - Tabela 1 mostra a porcentagem de joelhos em que o mesmo ou o próximo corte foi escolhido por ambos os observadores. Boa confiabilidade e concordância foram observadas entre as medições de TC e RM para medições de TT-ST e TP-STC, com um CCI de 0,774 (0,659$0,854, p<0,001)$ e $0,743(0,615-0,833, p<0,001)$, respectivamente. A distribuição é mostrada nos gráficos de BlandAltman (-Figs. 3 e 4). Observa-se a presença de $<6 \%$ das observações fora dos limites de concordância (intervalo de confiança [IC] de 95\%). As medições TT-ST e TP-STC foram espalhadas aleatoriamente perto do valor zero da diferença, e nenhum viés sistemático foi observado. As TT-ST médias na TC e na RM foram 17,1 $\pm 4,2 \mathrm{~mm}$ e $16,2 \pm 3,7 \mathrm{~mm}$, respectivamente. As distâncias médias TP-STC foram respectivamente $17,3 \pm 4,2 \mathrm{~mm}$ e $16,5 \pm 4,1 \mathrm{~mm}$. A confiabilidade entre avaliadores foi excelente para todas as medições (-Tabela 2).

\section{Discussão}

Nosso resultado mais importante foi a boa confiabilidade e concordância das medições TT-ST e TP-STC entre RM (usando a bobina de corpo) e TC. Uma vez que o grau de flexão do joelho influencia a rotação tibiofemoral e, portanto, as distâncias, ${ }^{16}$ a padronização rigorosa no posicionamento dos joelhos foi essencial para alcançar esse resultado. Quando a medição TT-ST é necessária, o estudo de TC pode ser substituído por 
86 A RM do joelho usando uma bobina corporal é equivalente à TC na medição da distância TT-ST Aivazoglou et al.

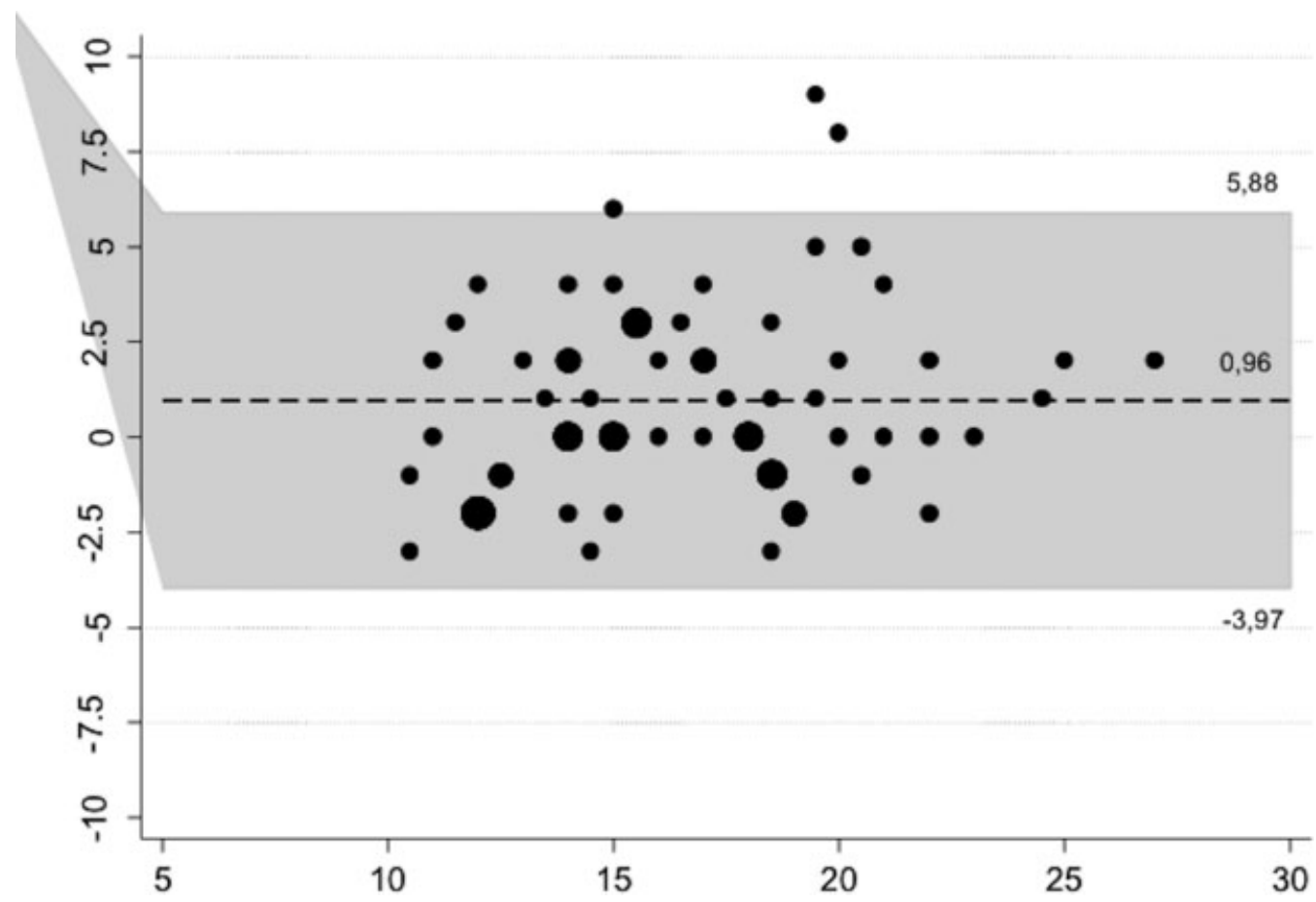

Fig. 3 O gráfico de Bland-Altman mostra as medições TT-ST espalhadas aleatoriamente dentro do intervalo de confiança. Apenas 4,4\% (3/68) dos casos estão fora dos limites da concordância.

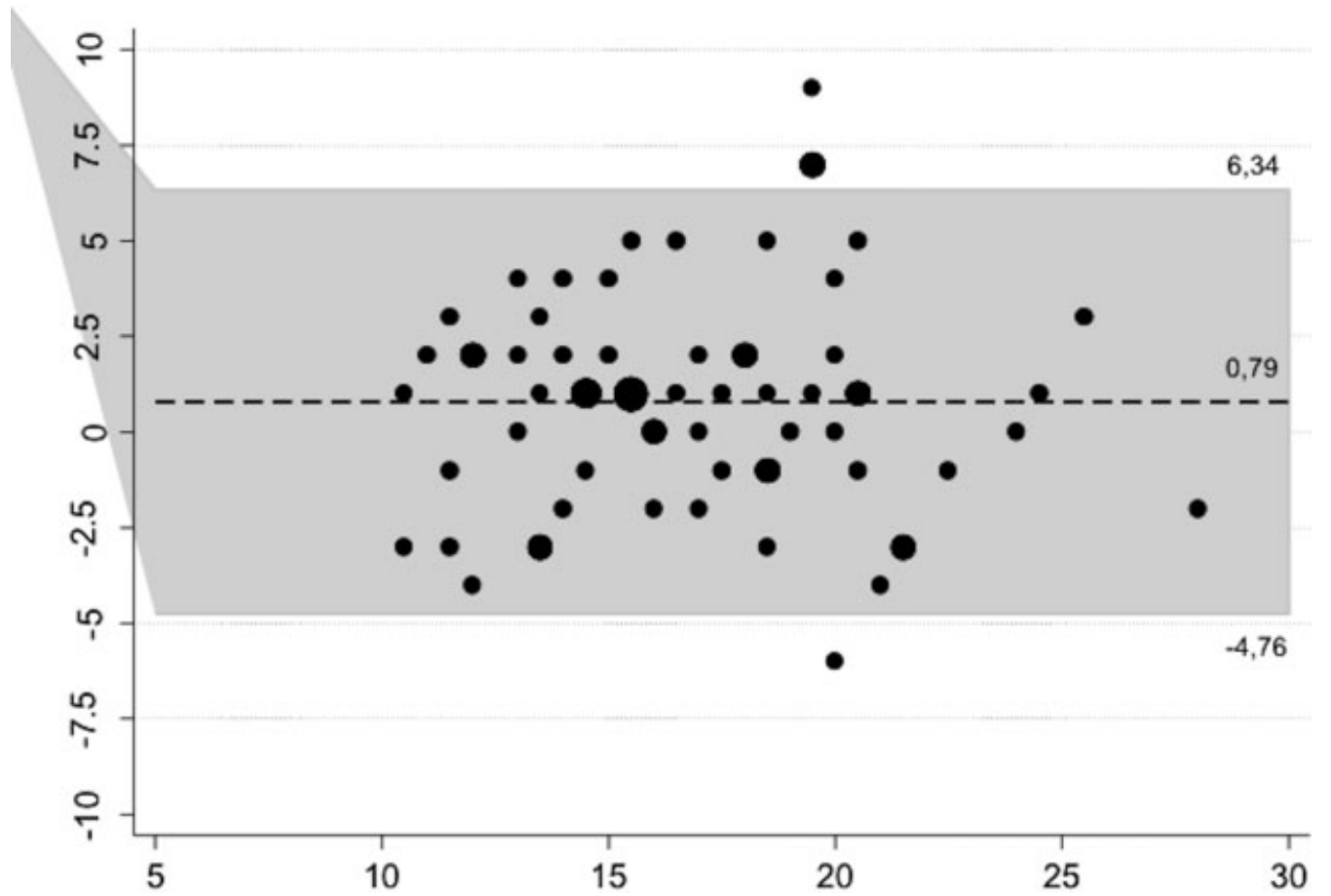

Fig. 4 O gráfico de Bland-Altman mostra as medidas TP-STC espalhadas aleatoriamente dentro do intervalo de confiança. Apenas 5,9\% (4/68) dos casos estão fora dos limites da concordância.

uma sequência axial ponderada em T1 dos joelhos usando a bobina de corpo, removendo a necessidade de exposição desnecessária à radiação neste cenário e, mais importante, permitindo o uso do mesmo limite $(15-20 \mathrm{~mm})$ usado classicamente em TC. Nosso estudo também confirma a excelente confiabilidade entre avaliadores das medidas de RM, que já havia sido mostrada em estudos anteriores. ${ }^{14,17,18}$
Schoettle et al. ${ }^{19}$ compararam TC e RM do joelho (com um protocolo de rotina do joelho) e encontraram uma excelente confiabilidade entre métodos, afirmando que tomografias adicionais não eram necessárias. No entanto, muitos estudos posteriores não foram capazes de reproduzir esses resultados, concluindo que as medidas de TC e RM TT-ST não são equivalentes, e que as medidas de RM são sistematicamente 
Tabela 2 Confiabilidade entre examinadores para medições de TC e RM

\begin{tabular}{|l|l|l|}
\hline & \multicolumn{2}{|l|}{ Coeficiente de correlação intraclasse } \\
\hline & (IC 95\%) & valor-p \\
\hline TC & & \\
\hline TT-ST & $0,872(0,760-0,934)$ & $<0,001$ \\
\hline TP-STC & $0,918(0,844-0,958)$ & $<0,001$ \\
\hline RM & & \\
\hline TT-ST & $0,833(0,693-0,912)$ & $<0,001$ \\
\hline TP-STC & $0,907(0,824-0,952)$ & $<0,001$ \\
\hline
\end{tabular}

Abreviações: IC, intervalo de confiança; RM, ressonância magnética; TC, tomografia computadorizada; TP-STC, distância tendão patelar-sulco troclear cartilaginoso; TT-ST; distância tubérculo tibial-sulco troclear.

menores, ${ }^{3,4,7-9}$ o que sugere que seria impreciso usar o mesmo limiar em RM e TC no diagnóstico e planejamento cirúrgico.

A distância TT-ST é altamente sensível a mudanças no posicionamento do joelho ${ }^{4,10}$ e, enquanto a TC é realizada com as pernas em extensão total, a bobina de joelho dedicada na RM envolve o joelho de uma forma que assume graus variáveis de flexão $\left(\sim 25^{\circ 7}\right)$ e desvio em varo. ${ }^{4}$ Uma posição parcialmente flexionada do joelho reduz as medições TT-ST ${ }^{7}$ devido à rotação interna progressiva da tíbia em relação ao fêmur durante a flexão. Seitlinger et al. ${ }^{16}$ estudaram a distância TT-ST em extensão e em diferentes graus de flexão em RM e descobriram que a distância TT-ST diminuiu significativamente durante a flexão em joelhos com instabilidade patelofemoral e em voluntários saudáveis. Aarvold et al. ${ }^{7}$ compararam a distância TT-ST em pacientes sintomáticos medida em estudos de RM usando uma bobina corporal para garantir a extensão total dos joelhos e na RM usando uma bobina de joelho dedicada, descobrindo que esta última subestima as medidas (diferença média: $8,6 \mathrm{~mm}$ ).

Em nenhum desses estudos, o posicionamento dos pés foi mencionado. Galland et al. ${ }^{20}$ realizaram os estudos de TC usando um dispositivo de apoio plantar para evitar a contração do quadríceps e, embora mencionassem a recomendação de colocar os pés no ângulo do degrau, consideraram que o posicionamento dos pés não afetaria as medidas femoropatelares (mas infelizmente não apresentaram dados para apoiá-lo). Decidimos padronizar o posicionamento dos pés por duas razões teóricas. Uma é a possibilidade de um indesejável alinhamento oblíquo da extremidade inferior examinada em relação ao eixo longitudinal da máquina e a segunda é que a gravidade atuando nos pés de um paciente deitado em decúbito dorsal poderia produzir um torque no joelho e rotação da tíbia em relação ao fêmur.

A seleção do corte pode ser uma fonte de discordância do TT-ST ou TP-STC final em ambos os métodos. Embora o CCI tenha sido excelente ou bom para todos os cortes, a concordância sobre o mesmo corte pode ser considerada ruim para a fatia 3 na TC $(32,4 \%)$ e na RM (52,9\%). Acreditamos que o longo diâmetro craniocaudal TT pode causar problemas para decidir qual corte escolher. Em relação ao uso de parâmetros ósseos ou de partes moles, embora ambos sejam confiáveis entre TC e RM, houve uma tendência de maiores coeficientes de correlação com o uso de parâmetros de partes moles, conforme observado nas medidas de RM de Wilcox et al. ${ }^{14}$ Esses resultados apontam para a recomendação de usar o TP como ponto de referência distal em vez de TT.

A única revisão sistemática e meta-análise sobre o tema ${ }^{5}$ sugere o uso de diferentes limiares para TC e RM $(15,5 \pm 1,5 \mathrm{~mm}$ para a distância TT-ST medida na TC e $12,5 \pm 2 \mathrm{~mm}$ para $\mathrm{RM})$, com a limitação de que não houve padronização do posicionamento e flexão dos joelhos e dos pontos de referência utilizados.

Ho et al. ${ }^{4}$ concluíram que estabelecer um posicionamento controlado e reprodutível do paciente seria vital para permitir a intercambialidade do uso de TC e RM na medição da distância TT-ST, e esse era o principal objetivo do nosso estudo.

Impedir o uso da TC neste cenário evitaria a exposição à radiação em uma população especialmente jovem, reduzindo assim seus riscos potenciais ao longo da vida e os custos gerais, embora adicionar uma sequência à RM do joelho aumentaria o tempo de estudo de RM.

As principais limitações de nosso estudo incluem uma amostra pequena e a avaliação exclusiva de voluntários assintomáticos. Pesquisas futuras devem avaliar a intercambialidade em pacientes com instabilidade patelar.

Outra limitação seria não podermos avaliar a importância isolada do posicionamento dos pés, visto que optamos por padronizar rigorosamente o posicionamento tanto dos joelhos quanto dos pés e não testamos diferentes posicionamentos dos pés.

Em conclusão, este foi o primeiro estudo que comparou a RM usando a bobina de corpo com a TC, considerada padrão ouro, na medição das distâncias TT-ST e TP-STC, com uma boa concordância entre esses métodos e uma excelente confiabilidade interexaminador, com a potencial implicação clínica de que a TC do joelho pode ser substituída por RM usando a bobina de corpo neste cenário clínico.

\section{Suporte Financeiro}

Não houve apoio financeiro de fontes públicas, comerciais, ou sem fins lucrativos.

\section{Conflito de Interesses}

Os autores não têm conflito de interesses a declarar.

\section{Agradecimentos}

Agradecemos ao Dr. Marcelo Astolfi Caetano Nico por dedicar o tempo e esforço necessários para revisar o manuscrito.

\section{Referências}

1 Thakkar RS, Del Grande F, Wadhwa V, et al. Patellar instability: CT and MRI measurements and their correlation with internal derangement findings. Knee Surg Sports Traumatol Arthrosc 2016;24(09):3021-3028

2 Waterman BR, Belmont PJ Jr, Owens BD. Patellar dislocation in the United States: role of sex, age, race, and athletic participation. J Knee Surg 2012;25(01):51-57 
3 Camp CL, Stuart MJ, Krych AJ, et al. CT and MRI measurements of tibial tubercle-trochlear groove distances are not equivalent in patients with patellar instability. Am J Sports Med 2013;41(08):1835-1840

4 Ho CP, James EW, Surowiec RK, et al. Systematic techniquedependent differences in CT versus MRI measurement of the tibial tubercle-trochlear groove distance. Am J Sports Med 2015;43(03):675-682

5 Tan SHS, Lim BY, Chng KSJ, et al. The Difference between Computed Tomography and Magnetic Resonance Imaging Measurements of Tibial Tubercle-Trochlear Groove Distance for Patients with or without Patellofemoral Instability: A Systematic Review and Meta-Analysis. J Knee Surg 2020;33(08):768-776

6 Smith TO, Davies L, Toms AP, Hing CB, Donell ST. The reliability and validity of radiological assessment for patellar instability. A systematic review and meta-analysis. Skeletal Radiol 2011;40 (04):399-414

7 Aarvold A, Pope A, Sakthivel VK, Ayer RV. MRI performed on dedicated knee coils is inaccurate for the measurement of tibial tubercle trochlear groove distance. Skeletal Radiol 2014;43(03):345-349

8 Hinckel BB, Gobbi RG, Filho EN, et al. Are the osseous and tendinouscartilaginous tibial tuberosity-trochlear groove distances the same on CT and MRI? Skeletal Radiol 2015;44(08):1085-1093

9 Anley CM, Morris GV, Saithna A, James SL, Snow M. Defining the Role of the Tibial Tubercle-Trochlear Groove and Tibial Tubercle-Posterior Cruciate Ligament Distances in the Work-up of Patients With Patellofemoral Disorders. Am J Sports Med 2015;43(06):1348-1353

10 Marquez-Lara A, Andersen J, Lenchik L, Ferguson CM, Gupta P. Variability in patellofemoral alignment measurements on MRI: Influence of knee position. AJR Am J Roentgenol 2017;208(05): 1097-1102

11 Zou GY. Sample size formulas for estimating intraclass correlation coefficients with precision and assurance. Stat Med 2012;31(29): 3972-3981
12 Ait-Ali L, Andreassi MG, Foffa I, Spadoni I, Vano E, Picano E. Cumulative patient effective dose and acute radiation-induced chromosomal DNA damage in children with congenital heart disease. Heart 2010;96(04):269-274

13 Dejour H, Walch G, Nove-Josserand L, Guier C. Factors of patellar instability: an anatomic radiographic study. Knee Surg Sports Traumatol Arthrosc 1994;2(01):19-26

14 Wilcox JJ, Snow BJ, Aoki SK, Hung M, Burks RT. Does landmark selection affect the reliability of tibial tubercle-trochlear groove measurements using MRI? Clin Orthop Relat Res 2012;470(08): 2253-2260

15 Koo TK, Li MY. A Guideline of Selecting and Reporting Intraclass Correlation Coefficients for Reliability Research. J Chiropr Med 2016;15(02):155-163

16 Seitlinger G, Scheurecker G, Högler R, Labey L, Innocenti B, Hofmann $\mathrm{S}$. The position of the tibia tubercle in $0^{\circ}-90^{\circ}$ flexion: comparing patients with patella dislocation to healthy volunteers. Knee Surg Sports Traumatol Arthrosc 2014;22(10):2396-2400

17 Pandit S, Frampton C, Stoddart J, Lynskey T. Magnetic resonance imaging assessment of tibial tuberosity-trochlear groove distance: normal values for males and females. Int Orthop 2011; 35(12):1799-1803

18 Sobhanardekani M, Sobhan MR, Nafisi Moghadam R, Nabavinejad S, Razavi Ratki SK. The Normal Value of Tibial Tubercle Trochlear Groove Distance in Patients With Normal Knee Examinations Using MRI. Acta Med Iran 2017;55(09):573-577

19 Schoettle PB, Zanetti M, Seifert B, Pfirrmann CWA, Fucentese SF, Romero J. The tibial tuberosity-trochlear groove distance; a comparative study between CT and MRI scanning. Knee 2006; 13(01):26-31

20 Galland O, Walch G, Dejour H, Carret JP. An anatomical and radiological study of the femoropatellar articulation. Surg Radiol Anat 1990;12(02):119-125 